\title{
Quantum Aspects of Electron-Light-Plasmon Interactions at the Atomic Scale
}

Javier García de Abajo ${ }^{1}$, Valerio Di Giulio ${ }^{2}$, Andrea Konečná ${ }^{3}$ and Vahagn Mkhitaryan ${ }^{2}$

${ }^{1}$ ICFO, Castelldefels, Catalonia, Spain, ${ }^{2}$ ICFO - The Institute of Photonic Sciences, Castelldefels, Catalonia, Spain, ${ }^{3}$ Materials Physics Center CSIC-UPV/EHU, San Sebastian, Navarra, Spain

Electron beams are ideal tools to controllably excite and probe plasmons and other nanoscale optical excitations with an unparalleled combination of space and energy resolutions. Spectroscopy performed through the analysis of electron energy loss and cathodoluminescence are widely used to obtain snapshots of these excitations. Additionally, access to the ultrafast sample dynamics is possible by recording photoelectrons excited with femtosecond light pulses, while several experiments demonstrate optical pumping followed by electron-beam probing with similar temporal resolution. In this talk, we will review recent advances in these techniques and present a unified theoretical description, along with several potential directions for improving the space-time-energy resolution and accessing quantum aspects of the samples [1].

As a first challenge, we will discuss fundamental limits to the coupling between electrons and optical excitations based on suitably tailored beam-electron wave functions, thus opening new directions for further increase in time resolution and the exploration of nonlinear phenomena with nanometer resolution [2]. Nonlinear optical interactions are be explored in the ultrafast regime, where prospects for sampling the nonlinear response with unprecedented combination of spatial and energy resolution are discussed [3], along with a physical scenario consisting of low-energy electrons undergoing strong interaction with the samples and entering the nonlinear optical response at the single_electron level [4]. Additionally, we will discuss recent theoretical results on fundamental aspects of the interaction of fast electrons with localized optical modes that are made possible by the noted advances. Using a quantum-optics description of the optical field, we predict that the resulting electron spectra strongly depend on the statistics of the sample excitations (bosonic or fermionic) and their population (Fock, coherent, or thermal), whose autocorrelation functions are directly retrieved from the ratios of electron gain intensities [5]. We further explore feasible experimental scenarios to probe the quantum characteristics of the sampled excitations and their populations.

\section{References}

1. A. Polman, M. Kociak, and F. J. García de Abajo, Nat. Mater. 18, 1158 (2019).

2. V. Di Giulio, V. Mkhitaryan, and F. J. García de Abajo, in preparation.

3. V. Di Giulio, M. Kociak, and F. J. García de Abajo, Optica 6, 1524 (2019).

4. J. Cox and F. J. García de Abajo, submitted.

5. A. Konečná, V. Di Giulio, V. Mkhitaryan, C. Ropers, and F. J. García de Abajo, arXiv:1912.01539. 